\title{
Study on the Iodine Reaction of Blood Neutrophiles in Cases of Lactating Mothers.
}

\author{
145th Report of the Peroxidase Reaction.
}

(92nd Human Milk Study.)

By

Kikuzo Isizaka.

(石 坂 籍造)

(From the Department of Pediatrics, Faculty of Medicine, Tohoku Imperial University, Sendai.

Director : Prof. A. Sato.)

\section{Introduction.}

According to Ogata, ${ }^{1)} \mathrm{Hos}$ okawa, ${ }^{2)} \mathrm{Hirai}^{3)}$ and $\mathrm{Asai}{ }^{4)}$ blood sugar content increases in B-avitaminosis. A decrease of the glycolytic enzyme in the blood of rice-diseased animals was proved by Gentile $^{5)}$ and Takayama, ${ }^{6)}$ and further Takemoto, ${ }^{7)}$ Sasaki, ${ }^{87}$ Ikebe, ${ }^{9)}$ Tiwaki, ${ }^{10)} \mathrm{Usuki}^{11)}$ and Era ${ }^{12)}$ confirmed that glycolysis in blood was disturbed in cases of B-avitaminosis or rice-disease. Thus, a disturbance of glycolysis will happen and a hyperglycemia. will result in the $\mathrm{B}$-avitaminotic body. Then, it is natural to deduce that an increase of glycogen content of leucocytes will occur in Bavitaminosis. This was confirmed by Miyakawa, ${ }^{13)}$ who saw an in-
1) T. Ogata, Nissin Igaku, $19231924,13,332$.
2) Sy. Hos okawa, Nihon Naikagakkai Zassi, 1924 1925, 12, 116.
3) R. Hiraj, Nihon Byorigakkai Zassi, 1921, 11, 80.
4) H. As a i, Nihon Naikagakkai Zassi, 1924 1925, 12, 736.
5) F. Gentile, Arch. di Fisiol., 1927, 25, 21.
6) S. Takay a ma, Tyugai Izi Simpo, 1925, No. 1078, 357.
7) H. Take mot o, Zikken Igaku Zassi, 1927, 11, 165.
8) K. Sa s aki, Nagasaki Igakkai Zassi, 1929, 7, 54.
9) K. I k e be, Nagasaki Igakkai Zassi, 1930, 8, 457.
10) Z. Tiwa ki, Nihon Seikwagakkai Kaiho, 1932, 7, 63.
11) Z. Us uki, Nagoya Igakkai Zassi, 1936, 43, 1119.
12) K. Era, Nagoga Igakkai Zassi, 1936, 44, 1091.
13) M. Mi y a a wa, Nagoya Igakkai Zassi, 1938, 47, 1077. 
crease of glycogen content of leucocytes in rice-diseased pigeons. If mothers secreting milk negative to A rakawa's reaction are, as has been shown by a number of reports ${ }^{14)}$ from our Laboratory, more or less in a state of $\mathrm{B}$-avitaminosis, then there will presumably be an increase of glycogen content of leucocytes in cases of Arakawa-negative mothers.

Erhlich's, ${ }^{15)}$ iodine reaction is a method which is used to examine an increase of glycogen content of leucocytes, because glycogen is an iodophilic substance. Ki yokaw a ${ }^{16)}$ published that Ehrlich's iodine reaction was remarkably positive in 6 cases out of 8 sucklings suffering from beriberi, the disease which is closely related to B-avitaminosis.

In the present treatise I described iodine reaction by the use of Ehrlich's method in the cases of Arakawa-positive and Arakawanegative mothers compared with the result of the reaction between these two groups of lactating mothers.

\section{Method of Investigation.}

1. Iodine reaction of blood neutrophiles :-

Kiyokawa's modification of Ehrlich's method was used. The technique is as follows :

14) T. A ra ka wa, Tohoku J. Exp. Med., 1930, 16, 118.

K. Suzuki and T. Arakawa, Ibid., 1930, 16, 228.

M. $\mathrm{C}$ h i ba and J. A be, Ibid, 1932, 19, 479.

M. Chiba, Ibid., 1932, 19, 284 and 486.

T. S u z u k i, Ibid., 1934, 23, 23.

T. Suzuki, Ibid., 1935, 25, 186.

T. S u zuki, Ibid., 1935, 25, 575 .

T. Suzuki, Ibid., 1937, 31, 280.

A. Takam ats u, Ibid., 1934, 23, 46 .

A. T a k a mat.s u, Ibid., 1934, 23, 372.

J. No z a k i, Ibid., 1934, 23, 60 .

T. Suzuki and A. T a k a mats u, Ibid., 1934, 24, 202.

T. S u z u i and A. Tak a mats u, Ibid., 1935, 25, 588.

J. K i m u ra, Ibid., 1934, 23, 494.

J. Kim u r a, Ibid., 1935, 27, 360.

J. K i m u r a, Ibid., 1935, 27, 374.

S. Sir a is i, Ibid., 1936, 28, 44.

S. Sir a is i, Ibid., 1937, 31, 268.

S. Sir a is i, Ibid., 1937, 31, 510 .

S. Sir a is i, Ibid, 1988, 32, 73 .

S. Sira is i, Ibid., 1938, 33, 60 .

H. 0 hta, and T. Suzuki, Ibid., 1937, 31, 247.

M. Is h i i, Ibid., 1937, 31, 580 .

15) Z. Ehrlieh, Zschr. t. klin. Medizin, 1883, 6, 33.

16) Y. Ki y kawa, Rinsyo Shonika Zassi, 1932, No. 9, 25. 
Blood was taken from an ear lobe of the mother. The blood film, dried in the air, is fixed for one minute on the copper plate heated over a flame. Then the fixed film is kept in a vapour of iodine in a vessel with many crystals of iodine on the bottom of it. The film is taken out from the vapour of iodine after 3 to 5 minutes and is mounted in cedar oil.

Neutrophiles are made objects of microscopic examination. The protoplasma of neutrophiles positive to the iodine reaction looks yellow or yellowish brown. I used signs to denote the intensity of the reaction in this paper. Sign $(t+)$ stood for neutrophiles entirely filled with brownish yellow granules, and sign $(+)$ for neutrophiles filled with yellow granules.

2. Arakawa's reaction of mother's milk :-

Milk was obtained from mothers of breast-fed infants and was examined with Arakaw a's reagent. The mothers were divided into three groups according to the intensity of A ra kaw a's reaction.

a. The normally or strongly Arakawa-positive group:

A rakawa's reaction of milk from both breasts was normally or strongly positive in one minute.

b. The weakly Arakawa-positive group: Arak awa's reaction of milk was of intermediate strength.

c. Arakawa-negative group: Arakawa's reaction of milk from both breasts was completely or almost completely negative after five minutes.

The different course of A rakawa's reaction during five minutes was shown by special signs (Cf. Table 1 ) for simplification.

Mothers who had been taking preparations of vitamin B, and those with normally positive Araka wa's reaction on one breast and completely negative Arakawa's reaction on the other breast were excluded in this treatise, because $I$ intend relating about such cases in subsequent reports.

\section{Results of Investigation.}

1. Iodine reaction of neutrophiles in the group of normally or strongly Arakawa-positive mothers (Cf. Table 2).

Fifty mothers belonged to this group and the iodine reaction was utterly negative in 37 cases (74\%). Even in 13 cases (26\%) showing a positive iodine reaction neutrophiles with $(t+)$ were not seen, while neutrophiles with $(+)$ were $8.8 \%$ in each case on an average. 


\section{Table 1.}

Table of signs for showing different A rakawa's reaction.





Explanation to the table:-

Take, for instance, the sign: $2(+t)$. This stands for Arakawa's reaction with the course $(H) 1^{\prime}(H) 2^{\prime}(H) 3^{\prime}(H) 4^{\prime}(H) 5^{\prime}$. The sign does not express prompt result of the reaction, so the prompt reaction of the sign: $2(H)$ may be $\left.(-) 0^{\prime}, \pm\right) 0^{\prime}$, $(+) 0^{\prime}$ or even $(H) 0^{\prime}$, but this will not matter much, as the result of the reaction in one minute is the most important.

2. Iodine reaction of the neutrophile in the group of weakly Arakawa-positive mothers* (Cf. Table 3).

There were 50 mothers belonging to this group and the iodine reaction was utterly negative in only 10 cases (20\%). Most of the cases i.e. 40 cases (80\%) showed a positive iodine reaction, and even the reaction of $(t+)$ was seen in 22 cases (44\%). Neutrophiles with

* "Arakawa-positive" may be used in two different senses. One of these is : Arakawa-positive in a biochemical sense. A sample of human milk is said to have become Arakawa-positive when it becomes blue on the addition of Arakawa's reagent. Here it means that the sample is not negative to Arakawa's reaction. The other of these two senses is: Arakawa-positive in a clinical sense. A sample of human milk is clinically Arakawa-positive only when it shows sucha reactions as $\#$ or W in one minute of the addition of A rak a a's reagent. Another sample of human milk may be Arakawa-positive in the first deseribed sense, but yet clinically negative. 
TABLE 2.

Iodine reaction of normally or strongly Aralcawa-positive group.

\begin{tabular}{|c|c|c|c|c|c|c|c|}
\hline \multirow{2}{*}{$\begin{array}{c}\text { Case } \\
\text { No. }\end{array}$} & \multirow{2}{*}{ Name } & \multirow{2}{*}{$\begin{array}{c}\text { Age } \\
\text { (years) }\end{array}$} & \multicolumn{2}{|c|}{ Arakawa's reaction } & \multicolumn{3}{|c|}{$\begin{array}{c}\text { Iodine reaction of blood } \\
\text { neutrophiles }\end{array}$} \\
\hline & & & Right & Left & $(-)$ & $(+)$ & $(H)$ \\
\hline 1 & S. 0 . & 26 & $1(H)$ & $I($ 坓) & 100 & 0 & 0 \\
\hline 2 & N. S. & 23 & $1(H)$ & $1(t+)$ & 100 & 0 & 0 \\
\hline 3 & U. S. & 29 & $1(+4)$ & $I(H)$ & 100 & 0 & 0 \\
\hline 4 & H. K. & 28 & $1(H)$ & $1($ H) & 100 & 0 & 0 \\
\hline 5 & T. $\mathbf{H}$ & 31 & $I(H)$ & $1(+4)$ & 100 & 0 & 0 \\
\hline 6 & $\mathbf{T} . \mathbf{K}$ & 27 & $I(+\#)$ & $1(H)$ & 100 & 0 & 0 \\
\hline 7 & H. A. & 22 & $1(t+1)$ & $1(4)$ & 100 & 0 & 0 \\
\hline 8 & N. I. & 27 & $1(\mathrm{~m})$ & $1(H)$ & 100 & 0 & 0 \\
\hline 9 & M. A. & 25 & $1(\#)$ & $1(4)$ & 100 & 0 & 0 \\
\hline 10 & K. K. & 23 & $1(++)$ & $1(+4)$ & 100 & 0 & 0 \\
\hline 11 & Y. T. & 24 & $I(H)$ & $1(+)$ & 88 & 12 & 0 \\
\hline 12 & T. $\mathrm{X}$. & 31 & $1(\pi)$ & $3(H)$ & 100 & 0 & 0 \\
\hline 13 & M. S. & 28 & $1(\#)$ & $1(H)$ & 100 & 0 & 0 \\
\hline 14 & Y. H. & 27 & $1(\#)$ & $2(+4)$ & 0 & 100 & 0 \\
\hline 15 & $\mathrm{R} . \mathrm{S}$ & 23 & $\mathbf{1}(H)$ & $1(H)$ & 100 & 0 & 0 \\
\hline 16 & U. M. & 31 & $1(H)$ & $1(H)$ & 100 & 0 & 0 \\
\hline 17 & T. $\mathbf{Y}$. & 36 & $1(H)$ & $1(H)$ & 100 & 0 & 0 \\
\hline 18 & M. M. & 26 & $1(+4)$ & $1(+)$ & 100 & 0 & 0 \\
\hline 19 & H. Y. & 40 & $3(H)$ & $1($ 井) & 100 & 0 & 0 \\
\hline 20 & H. S. & 31 & $3(H)$ & $1(\mathrm{HH})$ & 100 & 0 & 0 \\
\hline 21 & S. S. & 25 & $1(H)$ & $2(H)$ & 72 & 28 & 0 \\
\hline 22 & S. Y. & 25 & $4(H)$ & $1(H)$ & 10 & 90 & 0 \\
\hline 23 & A. S. & 25 & $2(++)$ & $2(H)$ & 100 & 0 & 0 \\
\hline 24 & Y. 0. & 26 & $2(H)$ & $2(+)$ & 100 & 0 & 0 \\
\hline 25 & H. K. & 36 & $4(H)$ & $1(+)$ & 84 & 16 & 0 \\
\hline 26 & K. M. & 30 & $1(H)$ & $4(H)$ & 80 & 20 & 0 \\
\hline 27 & M. A. & 42 & $3(H)$ & $I(+1)$ & 100 & 0 & 0 \\
\hline 28 & T. S. & 25 & $1(+\#)$ & $3(+)$ & 100 & 0 & 0 \\
\hline 29 & S. S. & 34 & $2(11)$ & $2(+4)$ & 100 & 0 & 0 \\
\hline 30 & K. T. & 23 & $2(+1)$ & $2(H)$ & 88 & 12 & 0 \\
\hline 31 & H. S. & 29 & $2(+1)$ & $2(+t)$ & 100 & 0 & 0 \\
\hline 32 & H. U. & 25 & $2(H)$ & $2(+1)$ & 100 & 0 & 0 \\
\hline 33 & $\mathbf{Y} . \mathbf{Y}$. & 28 & $1(H)$ & $3(+4)$ & 100 & 0 & 0 \\
\hline 34 & H. I. & 24 & $3(H)$ & $2(+1)$ & 80 & 20 & 0 \\
\hline 35 & U. M. & 25 & $5(H)$ & $2(H)$ & 100 & 0 & 0 \\
\hline 36 & T. S. & 26 & $3(H)$ & $5(H)$ & 100 & 0 & 0 \\
\hline 37. & T. H. & 39 & $3(+4)$ & $3(+1)$ & 100 & 0 & 0 \\
\hline 38 & T. S. & 28 & $4(H)$ & $3(4)$ & 100 & 0 & 0 \\
\hline 39 & M. $T$. & 27 & $3(t)$ & $3(+4)$ & 100 & 0 & 0 \\
\hline 40 & T. T. & 26 & $4(+)$ & $2(+1)$ & 100 & 0 & 0 \\
\hline 41 & Y. S. & 27 & $5(+1)$ & $2(+)$ & 80 & 20 & 0 \\
\hline 42 & K. S. & 25 & $2(H)$ & $4(H)$ & 90 & 10 & 0 \\
\hline 43 & S. S. & 23 & $2(+)$ & $3(+)$ & 100 & 0 & 0 \\
\hline 44 & M. Y. & 36 & $5(+)$ & $2(+)$ & 100 & 0 & 0 \\
\hline 45 & H. Y. & 25 & $3(H)$ & $4(+t)$ & 95 & 5 & 0 \\
\hline 46 & K. 0 . & 35 & $4(+t)$ & $4(H)$ & 8 & 92 & 0 \\
\hline 47 & K. K. & 25 & $3(H)$ & $2(H)$ & 100 & 0 & 0 \\
\hline 48 & K. K. & 35 & $4(H)$ & $4(H)$ & 85 & 15 & 0 \\
\hline 49 & S. E. & 31 & $4(H)$ & $4(H)$ & 100 & 0 & 0 \\
\hline 50 & S. E. & 30 & $6(+1)$ & $5(+1)$ & 100 & 0 & 0 \\
\hline
\end{tabular}

only $(+)$ were in most cases from $20 \%$ to $75 \%$ (on an average $55.7 \%$ ), and neutrophiles with $(+t)$ were in most cases from $20 \%$ to $80 \%$ (on 
TABLe 3.

Todine reaction of weakly Arakawa-positive group.

\begin{tabular}{|c|c|c|c|c|c|c|c|}
\hline \multirow{2}{*}{$\begin{array}{l}\text { Case } \\
\text { No. }\end{array}$} & \multirow{2}{*}{ Name } & \multirow{2}{*}{$\begin{array}{c}\text { Age } \\
\text { (years) }\end{array}$} & \multicolumn{2}{|c|}{ Arakawa's reaction } & \multicolumn{3}{|c|}{$\begin{array}{c}\text { Iodine reaction of blood } \\
\text { neutrophiles }\end{array}$} \\
\hline & & & Right & Left & $(-1)$ & $(+)$ & $(H)$ \\
\hline 1 & M. II. & 24 & $8(+)$ & $I(t)$ & 100 & 0 & 0 \\
\hline 2 & S. A. & 23 & $7(+)$ & $3(+4)$ & 92 & 8 & 0 \\
\hline 3 & H. N. & 39 & $3( \pm)$ & $1(H)$ & 68 & 32 & 0 \\
\hline 4 & H. S. & 26 & $1(H)$ & $1(-)$ & 84 & 26 & 0 \\
\hline 5 & S. T. & 31 & $4(+)$ & $3(+)$ & 100 & 0 & 0 \\
\hline 6 & M. K. & 33 & $5(+)$ & $5(+)$ & 92 & 8 & 0 \\
\hline 7 & T. N. & 25 & $5(+)$ & $4(+)$ & 0 & 100 & 0 \\
\hline 8 & Y. I. & 26 & $13( \pm)$ & $1(4)$ & 100 & 0 & 0 \\
\hline 9 & S. S. & 26 & $3( \pm)$ & $9(+)$ & 40 & 60 & 0 \\
\hline 10 & K. $\mathbb{Y}$. & 30 & $4(+1)$ & $12( \pm)$ & 0 & 0 & 100 \\
\hline 11 & T. $\mathrm{K}$. & 31 & $5(+)$ & $6( \pm)$ & 44 & 56 & 0 \\
\hline 12 & K. $\mathrm{s}$. & 26 & $6( \pm)$ & $8(+)$ & 100 & 0 & 0 \\
\hline 13 & N. I. & 27 & $2(+)$ & $5(-)$ & 16 & 84 & 0 \\
\hline 14 & M. T. & 30 & $7( \pm)$ & $5(+)$ & 100 & 0 & 0 \\
\hline 15 & K. s. & 29 & $5( \pm)$ & $5( \pm)$ & 100 & 0 & 0 \\
\hline 16 & T. $\mathrm{H}$. & 21 & $10( \pm)$ & $2( \pm)$ & 0 & 80 & 20 \\
\hline 17 & T. S. & 26 & $7( \pm)$ & $4( \pm)$ & 0 & 64 & 36 \\
\hline 18 & R. M. & 32 & $2(+)$ & $10(-)$ & 0 & 0 & 100 \\
\hline 19 & Y. M. & 29 & $14( \pm)$ & $7(+)$ & 64 & 36 & 0 \\
\hline 20 & $\mathrm{~T}, \mathrm{~K}$. & 34 & $11 \pm)$ & $8( \pm)$ & 12 & 76 & 2 \\
\hline 21 & M. H. & 31 & $6(-)$ & $4( \pm)$ & 15 & 70 & 15 \\
\hline 22 & T. Y. & 27 & $8( \pm)$ & $13( \pm)$ & 52 & 48 & 0 \\
\hline 23 & T. N. & 26 & $8(-)$ & $5(+)$ & 100 & 0 & 0 \\
\hline 24 & A. M. & 21 & $3(-)$ & $11 \pm$ & 25 & 75 & 0 \\
\hline 25 & M. 0 . & 30 & $15( \pm)$ & $9( \pm)$ & 100 & 0 & 0 \\
\hline 26 & M. T. & 33 & $15(\div)$ & $6( \pm)$ & 0 & 28 & 72 \\
\hline 27 & R. E. & 28 & $7(-)$ & $6( \pm)$ & 0 & 90 & 10 \\
\hline 28 & H. s. & 43 & $12( \pm)$ & $14 \pm$ & 0 & 100 & 0 \\
\hline 29 & K. K. & 21 & $14( \pm)$ & $13( \pm)$ & 0 & 100 & 0 \\
\hline 30 & H. H. & 31 & $7( \pm)$ & $11(-)$ & 0 & 0 & 100 \\
\hline 31 & T. T. & 29 & $4(+)$ & $16(-)$ & 50 & 50 & 0 \\
\hline 32 & M. K. & 29 & $12(-)$ & $4( \pm)$ & 100 & 0 & 0 \\
\hline 33 & S. M. & 30 & $6(-)$ & $6(-1)$ & 100 & 0 & 0 \\
\hline 34 & T. S. & 23 & $10( \pm)$ & $13(-)$ & 60 & 40 & 0 \\
\hline 35 & S. A. & 26 & $12(-)$ & $2(-)$ & 0 & 40 & 60 \\
\hline 36 & N. 0 . & 28 & $6(-)$ & $9(-)$ & 40 & 60 & 0 \\
\hline 37 & T. E. & 41 & $9( \pm)$ & $14(-)$ & 0 & 48 & 52 \\
\hline 38 & H. S. & 44 & $11( \pm)$ & $8(-)$ & 0 & 12 & 88 \\
\hline 39 & M. N. & 23 & $12( \pm)$ & $11(-)$ & 80 & 20 & 0 \\
\hline 40 & S. W. & 29 & $9( \pm)$ & $15(-)$ & 0 & 76 & 24 \\
\hline 41 & X. H. & 20 & $14( \pm)$ & $8(-)$ & 0 & 100 & 0 \\
\hline 42 & H. S. & 23 & $13( \pm)$ & $13(-)$ & 0 & 40 & 60 \\
\hline 43 & I. A. & 35 & $7( \pm)$ & $14(-)$ & 0 & 40 & 60 \\
\hline 44 & Y. M. & 32 & $7(-)$ & $7-3$ & 0 & 60 & 40 \\
\hline 45 & Y. S. & 22 & $1(-)$ & $16(-)$ & 0 & 0 & 100 \\
\hline 46 & N. K. & 26 & $15(-)$ & $3(-)$ & 0 & 90 & 10 \\
\hline 47 & T. T. & 23 & $15(-)$ & $12( \pm)$ & 0 & 20 & 80 \\
\hline 48 & Y. W. & 25 & $11( \pm)$ & $14(-)$ & 24 & 70 & 6 \\
\hline 49 & S. K. & 25 & $6(-)$ & $14(-)$ & 0 & 80 & 20 \\
\hline 50 & Y. T. & 29 & $16(-)$ & $4(-)$ & 0 & 88 & 12 \\
\hline
\end{tabular}

an average $45.8 \%$ ).

3. Iodine reaction of the neutrophile in the group of Arakawa- 
TABLE 4.

Iodine reaction of Arakawa-negative group.

\begin{tabular}{|c|c|c|c|c|c|c|c|}
\hline \multirow{2}{*}{$\begin{array}{l}\text { Case } \\
\text { No. }\end{array}$} & \multirow{2}{*}{ Name } & \multirow{2}{*}{$\underset{\text { (years) }}{\text { Age }}$} & \multicolumn{2}{|c|}{ Arakawa's reaction } & \multicolumn{3}{|c|}{$\begin{array}{l}\text { Iodine reaction of blood } \\
\text { neutrophiles }\end{array}$} \\
\hline & & & Right & Left & $(-)$ & $(+)$ & $(H)$ \\
\hline 1 & H. E. & 38 & $16(-)$ & $16(-)$ & 0 & 0 & 100 \\
\hline 2 & T. T. & 28 & $16(-)$ & $16(-)$ & 0 & 100 & 0 \\
\hline 3 & N. S. & 35 & $16(-)$ & $16(-)$ & 0 & 0 & 100 \\
\hline 4 & H. K. & 24 & $16(-)$ & $16(-)$ & 0 & 94 & 6 \\
\hline 5 & A. N. & 31 & $16(-)$ & $16(-)$ & 0 & 85 & 15 \\
\hline 6 & M. T. & 32 & $16(-)$ & $16(-)$ & 14 & 82 & 4 \\
\hline 7 & T. 0 . & 31 & $16(-)$ & $16(-)$ & 20 & 80 & 0 \\
\hline 8 & E. A. & 32 & $16(-)$ & $16(-)$ & 20 & 80 & 0 \\
\hline 9 & S. I. & 25 & $16(-)$ & $16(-)$ & 0 & 0 & 100 \\
\hline 10 & K. S. & 25 & $16(-)$ & $16(-)$ & 0 & 100 & 0 \\
\hline 11 & M. U. & 25 & $16(-)$ & $16(-)$ & 0 & 28 & 72 \\
\hline 12 & M. T. & 33 & $16(-)$ & $16(--)$ & 60 & 40 & 0 \\
\hline 13 & Y. S. & 25 & $16(-)$ & $16(-)$ & 0 & 0 & 100 \\
\hline 14 & T. A. & 20 & $16(-)$ & $16(-)$ & 0 & 64 & 36 \\
\hline 15 & K. M.' & 27 & $16(-)$ & $16(-)$ & 0 & ${ }^{\circ} 0$ & 100 \\
\hline 16 & N. S. & 30 & $16(-)$ & $16(-)$ & 0 & 0 & 100 \\
\hline 17 & S. S. & 35 & $16(-)$ & $16(-)$ & 0. & 15 & 85 \\
\hline 18 & T. S. & 21 & $16(-)$ & $16(-)$ & 10 & 90 & 0 \\
\hline 19 & M. K. & 27 & $16(-)$ & $I 6(-)$ & 0 & 0 & 100 \\
\hline 20 & Y. H. & 24 & $16(-)$ & $16(-)$ & 0 & 0 & 100 \\
\hline 21 & K. M. & 29 & $16(-)$ & $16(-)$ & 5 & 30 & 65 \\
\hline 22 & K. I. & 23 & $16(-)$ & $15(-)$ & 0 & 0 & 100 \\
\hline 23 & H. Y. & 27 & $16(-)$ & $16(-)$ & 0 & 0 & 100 \\
\hline 24 & A. T. & 23 & $15(-)$ & $15(-)$ & 0 & 10 & 90 \\
\hline 25 & T. Y. & 30 & $16(-)$ & $14(-)$ & 0 & 60 & 40 \\
\hline 26 & T. E. & 24 & $16(-)$ & $14(-)$ & 32 & 68 & 0 \\
\hline 27 & M. H. & 24 & $14(-)$ & $16(-)$ & 0 & 40 & 60 \\
\hline 28 & Y.A. & 28 & $14(-)$ & $16(-)$ & 35 & 65 & 0 \\
\hline 29 & H. S. & 30 & $14(-)$ & $16(-)$ & 0 & 0 & 100 \\
\hline 30 & T. E. & 25 & $15(-)$ & $15(-)$ & 0 & 0 & $\mathrm{~J} 0^{0}$ \\
\hline 31 & S. M. & 28 & $16(-)$ & $14(-)$ & 0 & 0 & 100 \\
\hline 32 & M. S. & 25 & $15(-)$ & $15(-)$ & 0 & 0 & 100 \\
\hline 33 & T. S. & 29 & $12(-)$ & $16(-)$ & 0 & 20 & 80 \\
\hline 34 & E. H. & 31 & $16(-)$ & $12(-)$ & 0 & 60 & $4^{0}$ \\
\hline 35 & N. 0 . & 23 & $16(-)$ & $14(-)$ & 0 & 35 & 65 \\
\hline 36 & T. M. & 20 & $14(-)$ & $16(-)$ & 17 & 33 & 50 \\
\hline 37 & X. K. & 30 & $8(-)$ & $16(-)$ & 0 & 74 & 26 \\
\hline 38 & Т. H. & 27 & $15(-)$ & $14(-)$ & 0 & 60 & $4^{0}$ \\
\hline 39 & M. 0 . & 24 & $12(-)$ & $16(-)$ & 0 & 80 & $2^{0}$ \\
\hline 40 & S. A. & 25 & $12(-)$ & $16(-)$ & 0 & 100 & 0 \\
\hline 41 & T. M. & 27 & $14(-)$ & $16(-)$ & 0 & 100 & 0 \\
\hline 42 & T. S. & 39 & $16(-)$ & $8(-)$ & 0 & 68 & 32 \\
\hline 43 & K. 0 . & 22 & $14(-)$ & $15(-)$ & 0 & 100 & 0 \\
\hline 44 & H. M. & 26 & $12(-)$ & $16(-)$ & 28 & 72 & 0 \\
\hline 45 & M. M. & 21 & $14(-)$ & $14(-)$ & 0 & 100 & 0 \\
\hline 46 & H. H. & 24 & $8(-)$ & $15(-)$ & 0 & 100 & 0 \\
\hline 47 & H. A. & $23^{\circ}$ & $12(-)$ & $12(-)$ & 0 & 0 & 100 \\
\hline 48 & H. S. & 35 & $12(-)$ & $8(-)$ & 37 & 63 & 0 \\
\hline 49 & H. 0 . & 25 & $8(-)$ & $8(-)$ & 15 & 85 & 0 \\
\hline 50 & H. S. & 27 & $16(-)$ & $16(-)$ & 0 & 100 & 0 \\
\hline
\end{tabular}

negative mothers (Cf. Table 4).

A total of 50 mothers belonged to this group, and there was no 
case showing negative iodine reaction. In most cases, i.e. in 33 cases ( $66 \%$ of all the cases) the iodine reaction of $(t+)$ was seen. Neutrophiles with $(t+)$ in most cases from $50 \%$ to $100 \%$ (on an average $70.5 \%$ ), and neutrophiles with $\left(t^{-}\right)$were in most cases from $40 \%$ to $90 \%$ (on an average $74.8 \%$ ).

\section{Comment.}

Of the normally or strongly Arakawa-positive mothers $74 \%$, and of the weakly Arakawa-positive mothers $20 \%$ showed negative iodine reaction of neutrophiles, but there was not a single case with the neutrophiles with negative iodine reaction in the group of Arakawa-negative mothers (Cf. Table 5). As to a positive iodine reaction of the neutrophile, on the contrary, none of normally Arakawapositive mothers showed the reaction of $(t+)$, but $44 \%$ of the weakly Arakawa-positive mothers and $66 \%$ of the Arakawa-negative mothers showed the reaction of $(t+)$ (Cf. Table 5 ). In other words, lactating women show an inverse proportion between their Arakawa's reac-

TABLE 5.

Distribution of the three different groups of lactating mothers according to the iodine reaction of neutrophiles.

\begin{tabular}{|c|c|c|c|}
\hline \multirow{2}{*}{$\begin{array}{c}\text { Iodine reaction } \\
\text { of } \\
\text { neutrophiles }\end{array}$} & \multicolumn{3}{|c|}{ Lactating mothers } \\
\hline & $\begin{array}{c}\text { Normally or strongly } \\
\text { Arakawa-positive } \\
\text { group }\end{array}$ & $\begin{array}{c}\text { Weakly Arakawa- } \\
\text { positive group }\end{array}$ & $\begin{array}{c}\text { Arakawa-negative } \\
\text { group }\end{array}$ \\
\hline- & $\begin{array}{l}37 \text { cases } \\
(74 \text { 90) }\end{array}$ & $\begin{array}{l}10 \text { cases } \\
(20 \%)\end{array}$ & $\begin{array}{l}0 \text { case } \\
(0 \%)\end{array}$ \\
\hline$+*$ & $\left.\begin{array}{rr}13 & n \\
26 & n\end{array}\right)$ & $\left.\begin{array}{rl}18 & n \\
(36 & n\end{array}\right)$ & $\begin{array}{l}17 \text { cases } \\
\left(\begin{array}{ll}34 \\
n\end{array}\right)\end{array}$ \\
\hline$H^{* * *}$ & $\begin{array}{l}0 \text { case } \\
\left(\begin{array}{ll}0 & n\end{array}\right)\end{array}$ & $\left.\begin{array}{rl}22 & \eta \\
44 & \eta\end{array}\right)$ & $\left(\begin{array}{cc}33 & n \\
66 & n\end{array}\right)$ \\
\hline $\begin{array}{r}\text { Foot note to } \\
\because \text { Cases } \\
\because \text { Cases }\end{array}$ & $\begin{array}{l}\text { hable:- } \\
\text { howing only the reac } \\
\text { howing the reaction }\end{array}$ & $\begin{array}{l}\text { n of }(+) \text {. } \\
(+t) \text {. }\end{array}$ & \\
\hline
\end{tabular}

tion and their iodine reaction of neutrophiles; the weaker the A ra$\mathrm{kaw}$ a reaction, the more intense the iodine reaction of neutrophiles is. This relation may also be shown by the following table (Cf. Table 6). Table 6 shows that the number of neutrophiles intensely positive to the iodine reaction increased as Arakawa's reaction became weaker. 
Table 6.

Average percentage of neutrophiles positive to the iodine reaction seen respectively in three groups of mothers.

\begin{tabular}{|c|c|c|c|}
\hline \multirow{2}{*}{ Group of mothers } & \multicolumn{3}{|c|}{ Neutrophiles positive to the iodine reaction } \\
\hline & $+*$ & $H * *$ & Total $(+$ and +$)$ \\
\hline $\begin{array}{c}\text { Normally or strongly Arakawa- } \\
\text { positive group }\end{array}$ & $8.8 \%$ & $0 \%$ & $8.8 \%$ \\
\hline $\begin{array}{c}\text { Weakly Arakawa-positive } \\
\text { group }\end{array}$ & 55.7 & 45.8 & $78.5 n$ \\
\hline Arakawa-negative group & 74.8 & 70.5 & 94.1 \\
\hline $\begin{array}{l}\text { Foot note to the table:- } \\
* \text { Cases showing only } \\
\text { * Cases showing the r }\end{array}$ & $\begin{array}{l}\text { reaction } \\
\text { ion of }(H\end{array}$ & & \\
\hline
\end{tabular}

Now, it became clear, also from my own experiment, that mothers secreting milk negative to Arakawa's reaction are abnormal or pathological on principle. Reports concerning Arak a wa's reaction from our Laboratory have shown, as stated in the introduction, that Arakawa-negative mothers are more or less in a state of B-deficit. If Kiyokawa's paper is taken into consideration, it is to be ascribed to B-avitaminosis that mothers with milk with negative ${ }^{\S}$ Ara$\mathrm{ka}$ a a's reaction show a positive iodine reaction of neutrophiles.

I shall publish in the near future a report upon the experimental demonstration of the relation between the iodine reaction of neutrophiles and B-deficit in lactating women.

\section{Conclusion.}

Mothers secreting milk negative to A rakawa's reaction show a positive iodine reaction of blood neutrophiles, which is probably a sign of B-avitaminosis.

\$ Weakly positive and completely or almost completely negative Arakawa's reaction. 